\title{
RESEARCHES OF LONG-TERM VARIATIONS IN URANUS AND NEPTUNE SPECTRA
}

\author{
Yu.Kuznyetsova $^{1}$, A.Vidmachenko ${ }^{1}$, O.Matsiaka ${ }^{2}$, Y. Shliakhetskaya ${ }^{1}$, M.Yushkin ${ }^{3}$, V.Krushevska ${ }^{1}$ \\ ${ }^{1}$ Main astronomical observatory of National Academy of Science of Ukraine, 27 Akademika \\ Zabolotnoho ave. 03680 Kyiv, Ukraine \\ ${ }^{2}$ Taras Shevchenko University of Kyiv, 64/13, Volodymyrska Street, Kyiv, Ukraine, 01601 \\ ${ }^{3}$ Special astrophysical observatory of Russian Academy of Sciences, Nizhnij Arkhyz, \\ Karachai-Cherkessian Republic, Russia 369167
}

ABSTRACT. The results of long-term spectral observations of Solar System giant planets Uranus and Neptune are presented here. Data were obtaining in period 2002-2012 at the peak Terskol Observatory (Northern Caucasus, Russia) using 2-meter Ritchey-Chretien telescope Zeiss-2000 with help coude echelle spectrograph and at the Special astrophysical observatory (SAO RAS, Northern Caucasus, Russia) with help 6-meter telescope using echelle spectrograph NES.

Raman light scattering of solar photons by hydrogen molecules in the atmosphere spectra of Uranus and Neptune was registered in most powerful Fraunhofer lines $\mathrm{H} \& \mathrm{~K}$ CaII as the "emission" details in center cores of these lines. Relative values of Raman scattering contribution for noted lines are estimated. Also, we have carried out long-term monitoring of Raman light scattering variations in cloudy surface spectra.

Key words: Uranus; Neptune; giant planets; Raman light scattering; Fraunhofer lines H\&K CaII.

\section{Introduction}

Raman scattering makes contribution to spectra because of prevailing content $\mathrm{H}_{2}$ molecules $(85 \%)$ in giant planet atmospheres (Cochran \& Trafton, 1978; Cochran, 1981). Raman effects in such powerful atmospheres arise in visual and UV spectral ranges. Our data were obtained in visual wavelength region. It is possible to register Raman effects only with high resolution and sufficient ratio signal/noise.

We have carried out long-term spectral observations of Solar System giant planets Uranus and Neptune. The main aims of this work are registration of Raman light scattering of solar photons by hydrogen molecules in the atmosphere spectra of Uranus and Neptune; relative value calculation of Raman scattering contribution to giant planet spectra in field of most powerful Fraunhofer lines H\&K Ca II; researching of possible long-time variations of Raman light scattering in spectra of Uranus and Neptune.

\section{Raman Light Scattering}

Raman light scattering can be described as incoherent nonresonant scattering of photons by molecules. The incident light which suffers the Raman scattering is just the solar spectrum. Raman light scattering is used as a diagnostic probe of planetary atmospheres because Raman spectra characterize the properties of molecules of the scattering medium (Cochran, 1981).

Raman scattering has an observable effects in planet spectra in UV and visual range. When the sunlight passes through the gas atmosphere of the planet Raman light scattering makes not deep "ghosts" in the spectrum of the scattered light (Wallace, 1972; Karkoschka, 1994). These ghosts have Rayleigh line profile and placed symmetrically on the left and on the right relatively absorption line. These shifted lines are called the Raman lines $\left(v_{R}\right)$. Each type of molecules makes own set of Raman lines:

$$
\begin{array}{ll}
v_{0} & - \text { Vibration frequency of incident light, } \\
v_{R} & - \text { Frequencies of shifted (Raman) lines } \\
\left(v_{0}-v_{R}\right)=\omega & - \text { Own frequency of molecule vibration } \\
v_{R}^{S}=v_{0}-\omega & - \text { Red (Stoks) «ghosts», } \\
v_{R}^{A}=v_{0}-\omega & - \text { Blue (antistoks) «ghosts». }
\end{array}
$$

Also Raman light scattering makes the pseudoemission features in center cores of solar lines. About $10 \%$ of the incident photons are being Raman scattering. Intensity of Fraunhofer lines in solar light reflected by planet atmosphere is less then in Solar spectrum. Therefore after exclusion of the solar spectrum in planet spectrum at the sites of the Fraunhofer lines H\&K CaII appear the "emission" details (pseudo-emission peaks). According to these works (Belton et al., 1973; Cochran \& Trafton, 1978; Karkoschka, 1994) the intensities of "emission" details in cores of H\&K CaII defined using low spectral resolutions can achieve 10-20\%.

\section{Observations and data processing}

Most of spectroscopic observations were carried out at 2-meter telescope of the peak Terskol Observatory (Northern Caucasus, Russia) with using high resolution coude echelle spectrograph [http://www.terskol.com/telescopes/maestro.htm] (Musaev, 1999; Kuznyetsova \& Bondar, 2002). Spectra were obtained during 2002-2012 in visible range about 3800-9000 Angstroms. Data for 2007 and 2008 year were obtained at 
Special astrophysical observatory (SAO RAS, Northern Caucasus, Russia) with help of 6-meter telescope using echelle spectrograph NES located at the Nasmyth-2 focus (1:30). Spectra were obtained in visible range about 3500-6000 Angstroms.

Spectral resolution $\mathrm{R}=45000$ for observations by both telescopes was used. Using such equipment it is possible to detect Raman light scattering by $\mathrm{H}_{2}$ in the atmosphere spectra of major planets. Catalog of obtained data are presented in Tab. 1 .

Solar type standard 16Cyg A was used as a comparison star for all data except Neptune (June 2002). For it was used solar type standard 35 Leo. Standard data processing were done using program pockets DECH95, DECH20T for Terskol data and MIDAS for SAO data.

\section{Results}

As mentioned above, Raman light scattering is the cause of appearance of pseudo-emission features in the Fraunhofer line center cores. This fact can be used as an excellent diagnostic probe of planetary atmospheres.

After exclusion of solar spectrum performed by division on the spectrum of solar-type standard, in spectra of Solar system giant planets at the sites of the Fraunhofer lines H\&K CaII appear the "emission" details (Morozhenko, 1997; Kuznyetsova \& Vid'machenko, 2005). So, we can obtain clear contribution of Raman light scattering in the region of cores H\&K CaII lines. Fig. 1 demonstrates these pseudo-emission peaks in cores of more powerful Fraunhofer lines H\&K CaII after exclusion solar spectrum.

We have estimated intensity values of pseudo-emission peaks placed in centers of solar H\&K CaII lines for Neptune and Uranus using our observational spectra. Results are presented in Tab. 2, Tab. 3.
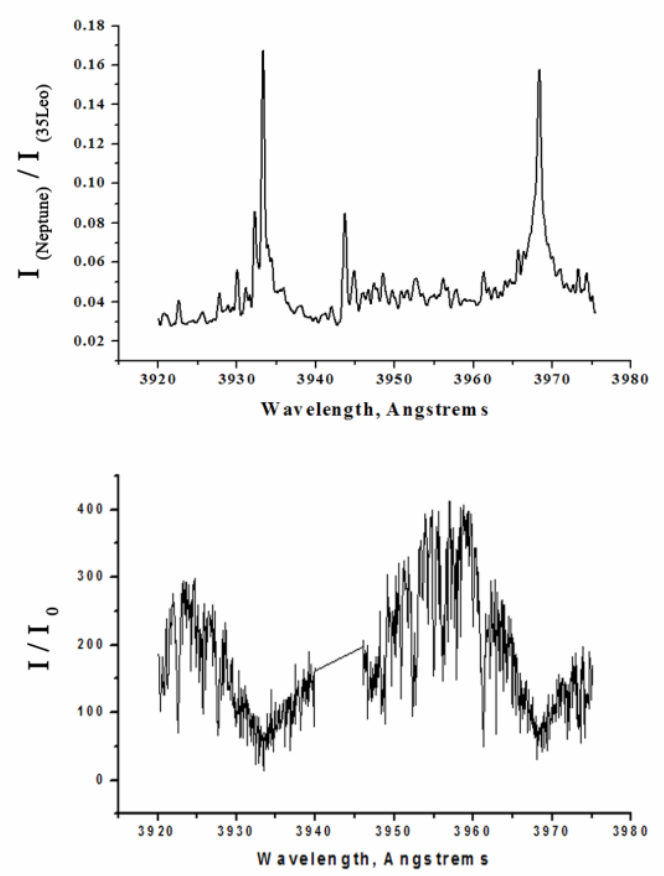

Figure 1: Spectrum of Neptune and the ratio spectrum Neptune/35 Leo in the vicinity of the Solar CaII H\&K lines (June 2002).

Uranus. Comparative variations of central intensities of Raman light scattering "emission" peaks in the field of lines H\&K CaII for 2007 and 2008 are shown at Fig. 2. Evaluations of central intensities of Raman light scattering "emission" peaks for Fraunhofer lines H\&K CaII are presented in Tab. 2. Relative intensity values of central pseudo-emission peaks have significant variations in the range $30-57 \%$. Probably, it can be explained due to the fact that Uranus was changing their position from 2007 to 2012 and different regions of cloudy surface fell into the field of view. Fig. 3 shows the positions for Uranus disks towards Earth for each observation date.

Table 1. Log of presented spectral data

\begin{tabular}{|c|c|c|c|c|}
\hline Object & Data & Observatory & $\begin{array}{c}\text { Number of } \\
\text { expositions }\end{array}$ & $\begin{array}{c}\text { Exposition } \\
\text { time, sec }\end{array}$ \\
\hline \multirow{4}{*}{ Uranus } & 30.09 .2007 & SAO, 6-m & 4 & 900 \\
\cline { 2 - 5 } & 13.08 .2008 & SAO, 6-m & 4 & 900 \\
\cline { 2 - 5 } & 01.12 .2008 & Terskol, 2-m & 2 & 2700 \\
\cline { 2 - 5 } & 11.10 .2011 & Terskol, 2-m & 2 & 1800 \\
\cline { 2 - 5 } & 29.11 .2012 & Terskol, 2-m & 2 & 1800 \\
\hline \multirow{4}{*}{ Neptune } & 10.06 .2002 & Terskol, 2-m & 2 & 3276 \\
\cline { 2 - 5 } & 30.09 .2007 & SAO, 6-m & 3 & 2400 \\
\cline { 2 - 5 } & 13.08 .2008 & SAO, 6-m & 5 & 2700 \\
\cline { 2 - 5 } & 02.12 .2008 & Terskol, 2-m & 2 & 3600 \\
\cline { 2 - 5 } & 11.10 .2011 & Terskol, 2-m & 2 & 3000 \\
\cline { 2 - 5 } & 21.11 .2012 & Terskol, 2-m & 2 & 2700 \\
\hline
\end{tabular}




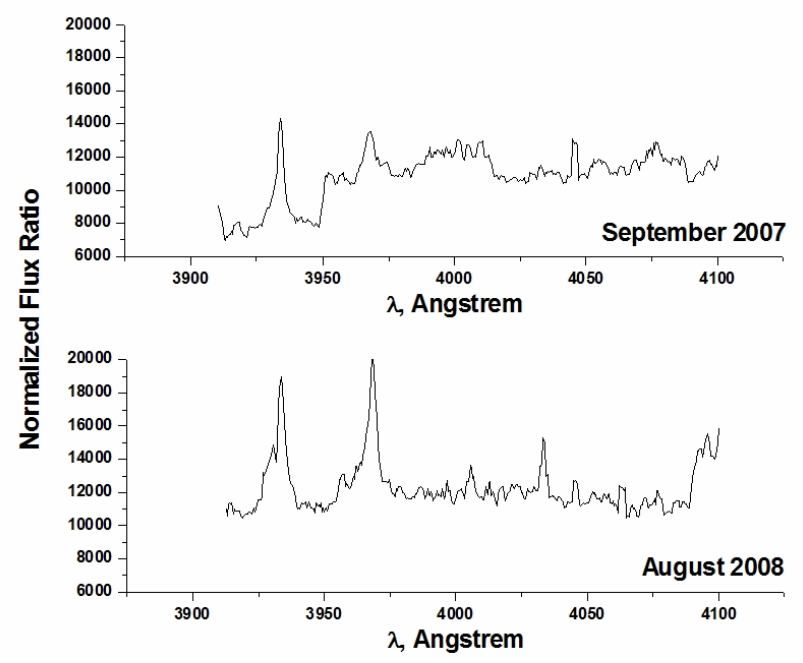

Figure 2: Raman scattering spectra of Uranus in the field of H\&K CaII lines.

Table 2. Evaluations of central intensities of Raman light scattering "emission" peaks for Fraunhofer lines H\&K Ca II

\begin{tabular}{|c|c|c|c|}
\hline Data & S/N & $\begin{array}{c}\text { H Ca II } \\
\mathbf{3 9 6 8 . 5} \AA\end{array}$ & $\begin{array}{c}\text { K Ca II } \\
\mathbf{3 9 3 3 . 7} \AA\end{array}$ \\
\hline September 2007 & 40 & $56 \%$ & $43.5 \%$ \\
\hline August 2008 & 40 & $57 \%$ & $43.2 \%$ \\
\hline December 2008 & 40 & $57 \%$ & $43.1 \%$ \\
\hline October 2011 & 40 & $47.6 \%$ & $30 \%$ \\
\hline November 2012 & 40 & $44.4 \%$ & $44 \%$ \\
\hline
\end{tabular}

Neptune. Comparative variations of central intensities of Raman light scattering "emission" peaks in the field of lines H\&K CaII for 2007 and 2008 are shown at Fig. 3. Relative intensity values of central pseudo-emission peaks in center cores of lines H\&K CaII for Neptune have less noticeable variations then for Uranus. They are in the range 40-50\% from 2007 to 2012. Estimations for 2002 show considerably smaller values because of low signal/noise relation. Fig. 5 shows Uranus was changing their position from 2007 to 2012. Evaluations of central intensities of Raman light scattering "emission" peaks for Fraunhofer lines H\&K Ca II are presented in Tab. 3.

Table 3. Evaluations of central intensities of Raman scattering "emission" peaks for Fraunhofer lines H\&K Ca II

\begin{tabular}{|c|c|c|c|}
\hline Data & S/N & $\begin{array}{c}\text { H Ca II } \\
\mathbf{3 9 6 8 . 5} \AA\end{array}$ & $\begin{array}{c}\text { K Ca II } \\
\mathbf{3 9 3 3 . 7} \AA\end{array}$ \\
\hline June 2002 & 15 & $25.4 \%$ & $10.7 \%$ \\
\hline September 2007 & 40 & $46.5 \%$ & $40.1 \%$ \\
\hline August 2008 & 40 & $42 \%$ & $40 \%$ \\
\hline December 2008 & 30 & $40 \%$ & $40 \%$ \\
\hline October 2011 & 40 & $45 \%$ & $42 \%$ \\
\hline November 2012 & 30 & $50 \%$ & $40.2 \%$ \\
\hline
\end{tabular}
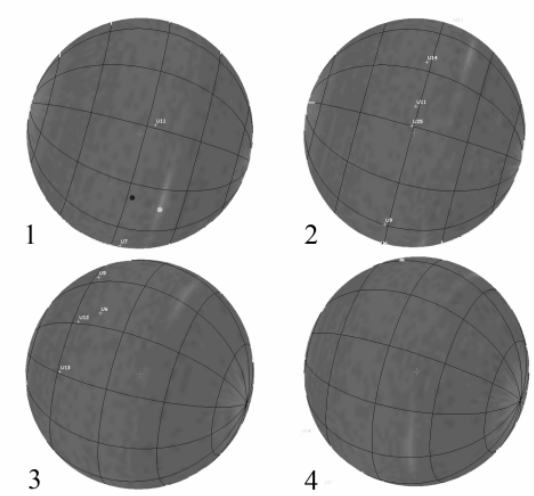

Figure 3: Positions of Uranus towards Earth for observation dates: 1-2007, 2-2008, 3-2011, 42012.

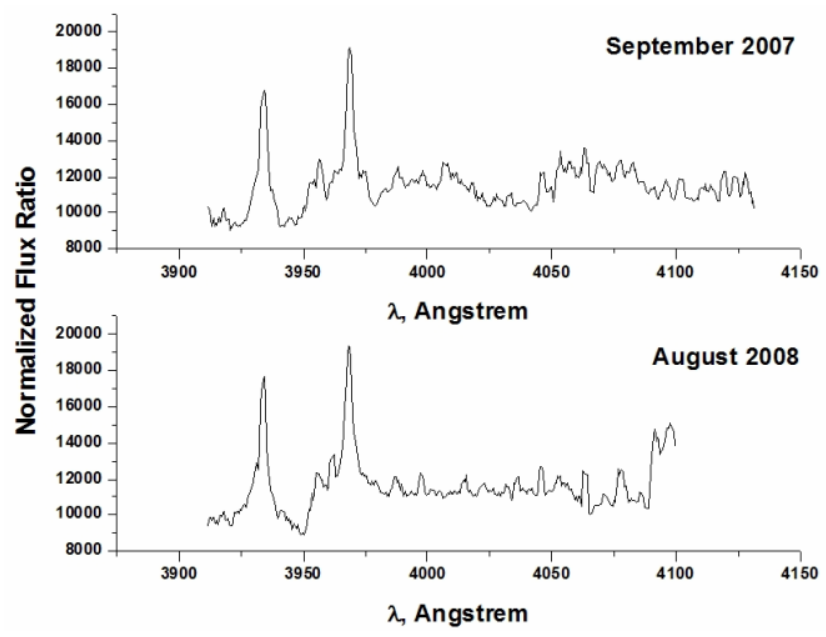

Figure 4: Raman scattering spectra of Uranus in the field of H\&K CaII lines.

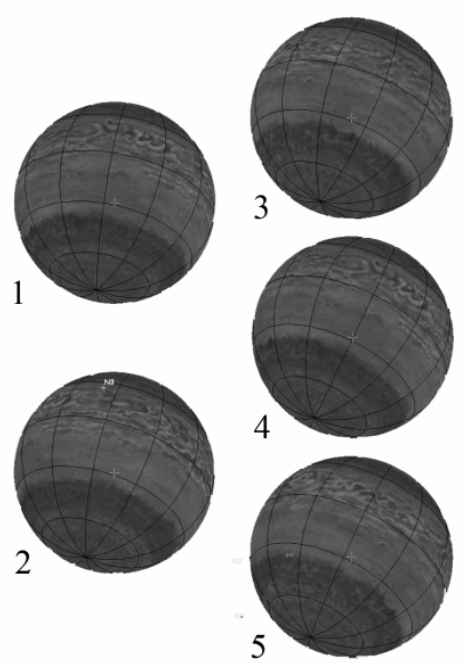

Fig.5. Positions of Neptune towards Earth for observation years: 1-2002; 2-2007, 3-2008, 4-2011; 5-2012. 


\section{Conclusions}

So, based on the long-term spectral observations of Solar system giant planets Uranus and Neptune, we have got such results:

1. Raman scattering is registered for all Uranus and Neptune spectra obtained from 2002 to 2012 years.

2. Estimates of clear Raman light scattering contribution in central cores of $\mathrm{H} \& \mathrm{~K}$ Ca II are changed in the range $10-56 \%$. Such high values compared with results of other researches can be explained by the high spectral resolution ( $\mathrm{R}=45000)$ of observations.

3. Reasons of variations of Raman scattering values can be aerosol clouds in planet atmosphere placed on differ heights. These clouds change the depth of gas column and respectively the values of Raman light scattering are vary.

4. "Ghosts" of H\&K CaII lines were not registered due to insufficient level of ratio Signal/Noise.

\section{References}

Belton M.J.S., Wallace L., Price M.J.: 1973, Astrophys. J., 184, №3, 143.

Cochran W.D., Trafton L.M. et al.: 1978, Astrophys. J., 219, 756.

Cochran W.D.: 1981, Adv. Space Res., 1, 143.

Karkoschka E.: 1994, Icarus, 111, N 1, 174.

Kuznyetsova Y., Bondar A.: 2002, J. Phys. Studies, 6, N4, 411.

Kuznyetsova Y., Vid'machenko A.: 2005, Kinematics and Physics of Celestial Bodies, Suppl. Ser., N 5, 480.

Morozhenko A.: 1997, Kinematics and Physics of Celestial Bodies, 13, N 4, 22.

Musaev F., Galazutdinov G., Sergeev A. et al.: Kinematics and Physics of Celestial Bodies, 1999, 15, N 3, 282.

Wallace L.: 1972, Astrophys. J., 176, 249. 